\title{
A Rare Case of Candida Tropicalis Infection of a Total Hip Arthroplasty: A Case Report and Review of Literature
}

\author{
A Azam, MRCS, PK Singh*, MRCS, VK Singh**, MRCS, A Siddiqui***, BDS \\ Orthopaedics, Manchester Royal Infirmary, Manchester, UK \\ * Neurovascular Surgery/ Medical Physics, Royal Hallamshire Hospital, Sheffield, UK \\ ** Orthopaedics, Luton and Dunstable Hospital, Luton, UK \\ *** Dental Sciences, Buddha Institute of Dental Sciences, Patna, India
}

\begin{abstract}
Fungal prosthetic joint infection is rare with Candida albicans being the most frequently reported pathogen in medical literature. We report here a rare case of Candida tropicalis infection affecting a total hip arthroplasty in a 73y male that failed to heal with initial treatment antibiotics and was eventually successfully treated with serial debridement, removal of prosthesis and antifungal medications. Revision of the total hip arthroplasty was performed four months after the patient was deemed infection free. In most cases, removal of implants, thorough debridement, and effective antifungal therapy are necessary for the eradication of infection. Reimplantation of the prosthesis can then be performed successfully in a staged surgical procedure. Orthopaedic surgeons should consider the possibility of a fungal infection in prosthetic joints especially in immune compromised patients especially if the infection is persistent or does not follow the expected course after treatment.
\end{abstract}

Key Words:

Candida tropicalis, Prosthetic joint infection, Antifungal therapy

\section{INTRODUCTION}

Infection is the second most common cause of prosthetic joint failure ${ }^{1}$. The potential for the morbidity and cost associated with repeat surgery, prolonged medical treatment and joint immobilization render importance to the accurate, timely diagnosis, and appropriate treatment of prosthetic joint infection (PJI). The actual incidence of fungal infection is not known, but is estimated to be about $1 \%$ of all $\mathrm{PJI}^{2-3}$. Candida albicans is the most commonly reported Candida fungal prosthetic joint infections. Conventional treatment of fungal prosthetic joint infection usually includes surgery followed by a long period of antifungal medication. Only two cases of Candida Tropicalis prosthetic joint infection have been mentioned in the literature to date ${ }^{3}$. We report here a case of total hip replacement infected with this rare organism that was successfully treated with antifungal pharmacotherapy and excision arthroplasty.

\section{CASE REPORT}

A 73-year-old man, who had a bilateral total hip replacement nine years earlier, was admitted to our hospital with a oneday history of fever and severe pain in his right hip. There was no recent history of injury around the hip and the only other significant clinical finding was a diabetic ulcer on the right great toe. Associated co-morbidities were insulin dependent diabetes and chronic renal failure. The patient's condition deteriorated very quickly over the next two days and he was transferred to an intensive care unit when he became septic. Upon transfer to the ICU, the patient had a very high white cell count (20.4x109/litre) and CRP was 307. He was started on intravenous vancomycin (1 gm once daily) for the sepsis along with adrenaline support. At that time, an ultrasound scan of the right hip was negative for any effusion, and a CT scan of abdomen, pelvis and hips was also normal. Two days later, a bone scan was requested which showed no signs of sepsis in the pelvis and hips but was positive in the area of the right great toe. On the third day, debridement of the right great toe was performed. At that time, right hip was aspirated and revealed frank pus (approximately $20 \mathrm{ml}$ ) and that was followed by proper hip wash out. The right hip wound swab grew gram negative bacilli and gram positive cocci, and on extended culture, mixed coliform and proteus were isolated. The antibiotics were changed to intravenous piperacillin/tazobactum ( $4.5 \mathrm{gm}$ twice daily) and oral rifampicin ( $450 \mathrm{mg}$ twice daily), as per sensitivities identified by the staff microbiologist.

Patient had to undergo five further debridements of the right hip and toe. The right toe was eventually amputated due to development of gangrene. In spite of repeated debridements of hip, the wound failed to heal. Histology was then suggestive of PJI. Further culture of the right hip capsule and acetabular membrane grew Candida tropicalis that showed sensitivity to caspofungin and fluconazole. The 


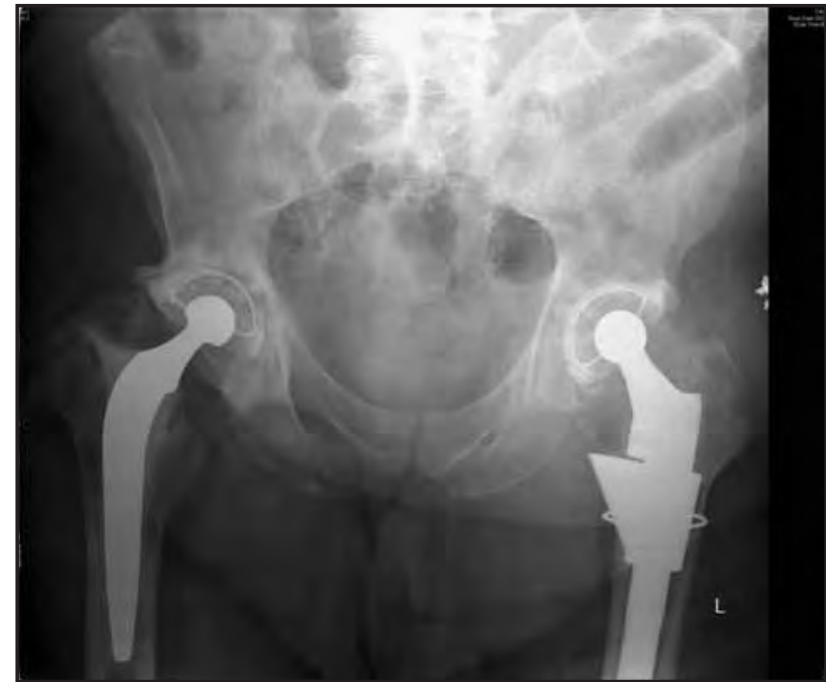

Fig. 1: Plain anterolateral radiograph of pelvis with both hips at the time of admission, showing loosening the hip prosthesis on the right side

patient was therefore started on caspofungin, which was changed to fluconazole after one week. Six weeks later, the prosthesis was removed and a Palacos cement spacer was inserted. After eight weeks of fluconazole treatment the wound healed well without no signs of infection and further cultures were sterile. Inflammatory markers also improved. Four months later as the patient was still infection free, a second stage revision of right hip was performed through an incision over the previous scar (using an S-ROM prosthesis). A tissue sample at the time of revision surgery were sent for microbiology and showed no growth. The patient was discharged one week following surgery and remained symptom free with no signs of local or systemic infection at twelve months follow-up.

\section{DISCUSSION}

Prosthetic joint infections are extremely difficult to treat, and in most of cases, removal of infected prosthesis is required to eradicate the infection ${ }^{4}$. Diagnosis of prosthetic-joint infection (PJI) is difficult, because the infecting agent would probably not be present in synovial fluid samples due to its exclusive presence as a device-associated biofilm ${ }^{5}$. Resistance to natural host defence mechanisms and to most antibiotics further complicates treatment ${ }^{5}$.

Darouiche et al. ${ }^{3}$ reviewed 10 cases of Candidal prosthetic joint infections and found that Candida albicans was the offending organism in four patients, Candida Parapsilosis in three, Candida Tropicalis in two, and Candida (torulopsis) glabrata in one. All patients were treated with removal of the prosthesis and antifungal therapy. Replacement with a new prosthetic joint was attempted in only two cases and successful in only one. Phelan et al. ${ }^{2}$ described that delayed

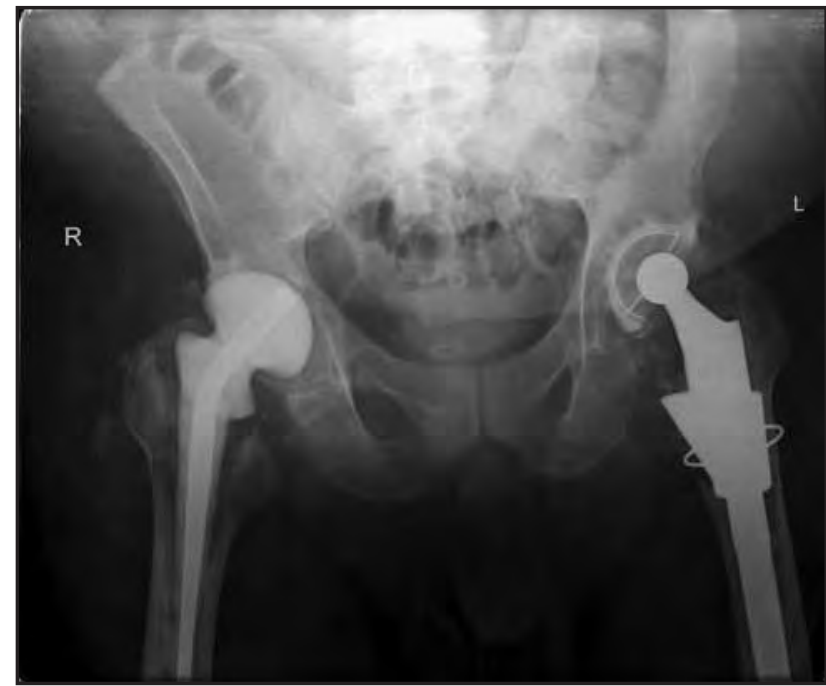

Fig. 2: X-ray pelvis with both hips AP view taken after insertion of Palacos cement spacer that followed removal of right total hip prosthesis

reimplantation arthroplasty for Candidal prosthetic joint infection is possible after completion of appropriate antifungal therapy.

In most cases, removal of infected prosthesis is required; in fact, Tattevin et al. ${ }^{6}$ reviewed 69 cases of hip and knee prosthetic infection and found that only 13 cases that had symptoms of shorter duration that did not require further surgical debridement. They concluded that debridement with retention of prosthesis is rarely sufficient prosthetic to fully eradicate the joint infection and this therapeutic approach should be considered only when the duration of symptoms is very short.

Prenzel et al. ${ }^{7}$ described a case of prosthetic joint infection, initially treated with antibiotics and surgical debridement without any success. Cultures later revealed Staphylococcus aureus, Enterococcus, and Candida parapsilosis. After removal of the prosthesis, cement spacer insertion and fluconazole treatment, the infection was controlled. Our case was quite similar in that it did not respond to antibiotic therapy and serial debridement but quickly responded to antifungal treatment at which time the wound healed nicely.

Due to the limited number of reported cases, firm recommendations for optimal treatment of Candidal joint infections are not yet available, although fluconazole has been widely used. Penk et al. ${ }^{8}$ reviewed the use of fluconazole in 32 patients and found that the mean duration of therapy was 6 months (maximum, 2 years) with an average dose of 200-400 mg/day (maximum, $800 \mathrm{mg} /$ day). They concluded that fluconazole was effective and safe in acute therapy alone or in combination with surgery as well as in long term suppression therapy. 
Girdlestone arthroplasty has been considered the standard surgical treatment of the prosthetic joint infections ${ }^{2}$. After resection arthroplasty, delayed reimplantation is usually performed with favourable results ${ }^{9}$. Indeed, we treated our patient successfully with fluconazole, removal of prosthesis and insertion of a Palacos cement spacer, followed by second stage revision. The microbiologist and pharmacist initially advised caspofungin because the patient was on rifampicin and, concurrent administration of rifampicin reduces the effect of fluconazole. After the rifampicin was stopped, the patient was switched over to Fluconazole. Caspofungin is considered a restricted drug and wherever possible fluconazole should be used for initial empirical therapy.
Fungal aetiology, although quite uncommon, should be considered in the differential diagnosis of PJIs especially in those not responding to conventional antimicrobials. Awareness of the extremely rare type of fungal PJI reported here is prudent. Removal of prosthesis remains a time proven decision in most of the cases, and should be accompanied by appropriate antifungal therapy. The need for a debridement should be assessed in each individual case. Late recurrence is common in bone and joint infection and a too early assumption of nonrecurrence of the infection is a therapeutic pitfall. A long infection free period is of utmost importance before embarking upon reimplantation. A staged arthroplasty and long term antifungal treatment seem to be most appropriate to reduce the risk of infection recurrence. 


\section{REFERENCES}

1. Anguita-Alonso P, Hanssen AD, Patel R. Prosthetic joint infection. Expert Rev Anti Infect Ther 2005 Oct;3(5):797-804.

2. Phelan DM, Osmon DR, Keating MR, Hanssen AD. Delayed reimplantation arthroplasty for candidal prosthetic joint infection: a report of 4 cases and review of the literature. Clin Infect Dis 2002 Apr 1; 34(7): 930-8.

3. Daurouiche RO, Hamill RJ, Musher DM, Young EJ, Harris RL. Periprosthetic candidal infections following arthroplasty. Rev Infect Dis 1989 11: 89, 1989.

4. Steckelberg JM, Osman DR. Prosthetic joint infection. In: Bisno AL, Waldvogel FA, editors. Infection associated with indwelling medical devices 3rd ed. Washington, DC: ASM Press, 2000: 173

5. Zimmerli W. Infection and musculoskeletal conditions: Prosthetic-joint-associated infections. Best Pract Res Clin Rheumatol 2006 Dec; 20(6): 1045-63.

6. Tattevin P, Crémieux AC, Pottier P, Huten D, Carbon C. Prosthetic joint infection: when can prosthesis salvage be considered? Clin Infect Dis. 1999 Aug; 29(2) :292-5.

7. Prenzel KL, Isenberg J, Helling HJ, Rehm KE. Candida infection in hip alloarthroplasty. Unfallchirurg 2003 Jan; 106(1): 70-2.

8. Penk A, Pittrow L. Fungal arthritis-a rare complication of systemic candidiasis or orthopedic intervention. Review of therapeutic experience with Fluconazole. Mycoses 1998; 41 Suppl 2: 45-8.

9. Su JK, Huang TL, Lin SY. Total knee arthroplasty in tuberculous arthritis. Clin Orthop 323: 181, 1996. 